\title{
A Late Dermatologic Presentation of Bullous Pemphigoid Induced by Anti-PD-1 Therapy and Associated with Unexplained Neurological Disorder
}

\author{
Xiaoxiao Wang ${ }^{a}$ Mariano Suppa ${ }^{b, c}$ Pascal Bruderer $^{d}$ Nicolas Sirtaine ${ }^{e}$ \\ Sandrine Aspeslagh ${ }^{f}$ Joseph Kerger ${ }^{a}$ \\ aDepartment of Medical Oncology, Institut Jules Bordet, Université Libre de Bruxelles,

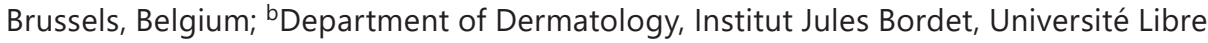 \\ de Bruxelles, Brussels, Belgium; 'Department of Dermatology, Hôpital Erasme, Université \\ Libre de Bruxelles, Brussels, Belgium; ${ }^{\mathrm{d} D e p a r t m e n t}$ of Dermatology, Centre Hospitalier \\ Universitaire Saint-Pierre, Université Libre de Bruxelles, Brussels, Belgium; eDepartment \\ of Pathology, Institut Jules Bordet, Université Libre de Bruxelles, Brussels, Belgium; \\ fDepartment of Medical Oncology, Universitair Ziekenhuis Brussel, Vrije Universiteit Brussel, \\ Brussels, Belgium
}

\section{Keywords}

Anti-PD-1 antibody · Anti-PD-L1 antibody · Bullous pemphigoid · Immunotherapy ·

Melanoma $\cdot$ Neurological disorder

\begin{abstract}
Immunotherapy has become the standard of care for various cancer types. The widespread use of immune checkpoints inhibitors confronts us with a whole range of novel immune-related adverse events. Skin toxicity is one of the most frequent adverse events. In this article, we report a case of anti-PD-1 induced late bullous pemphigoid (BP) with mucosal erosions and associated with a troublesome neurological disorder of undetermined origin in a patient with metastatic melanoma. Skin biopsy was essential to make the diagnosis and rapid initiation of systemic prednisolone played a role in favorable clinical outcome of BP. We will discuss the difficulty of early diagnosis of BP, its unusual association with neurological disorders, and the specific management of this particular dermatological entity.
\end{abstract}




\section{Introduction}

The advent of immunotherapy and particularly of immune checkpoint inhibitors (ICIs) has led to major advances in the treatment of cancers in recent years. By blocking pathways of inhibition of immune system, ICIs using anti-CTLA-4 (cytotoxic T lymphocyte-associated protein 4) antibodies, anti-PD-1 (programmed death-1) and anti-PD-L1 (programmed death-ligand 1) antibodies enable the immune system to recognize cancer cells and to proceed to their elimination through cytotoxic T-cell activation [1]. Currently, anti-PD-1 antibodies such as pembrolizumab and nivolumab are approved for the treatment of several cancers, but not limited to locally advanced and metastatic melanoma, nonsmall-cell lung cancer, head and neck squamous cancer, urothelial carcinoma, gastric adenocarcinoma, mismatch-repair-deficient solid tumors, Hodgkin lymphoma, hepatocellular carcinoma, and renal cell carcinoma [2].

Immune-related adverse events (irAEs) can affect multiple organs and resemble several known autoimmune syndromes. Skin toxicities are reported in $30-50 \%$ of patients treated with ICIs but are less frequently reported with anti-PD- 1 antibodies than with anti-CTLA-4 antibodies. The most common dermatologic irAEs are rash, pruritus, and vitiligo [3]. Due to the widespread use of ICIs, even rare side effects might become more prevalent. Therefore, bullous disorders have been reported in approximately $1 \%$ of patients treated with antiPD-1/PD-L1 [4]. Emerging case reports and series of cases have described bullous pemphigoid (BP) as a dermatologic irAE [5-14]. Autoimmune mechanisms underlying classic BP are well described, but exact mechanisms of anti-PD-1/PD-L1 induced BP and its association with neurological disorders are much more unclear $[15,16]$. The recognition and adequate treatment are thus essential for maintaining the quality of life of cancer patients.

\section{Case Presentation}

A 70-year-old patient was admitted to our outpatient clinic to receive the 35 th administration of Pembrolizumab in the context of a metastatic melanoma.

The primary melanoma BRAF wild-type of unknown stage had been surgically removed from the scalp in 2013. Two years later, he presented with a solitary lung metastasis and a brain relapse that was treated by surgical resection followed 3 months later by whole-brain radiotherapy on relapse with several new metastatic brain lesions and a first-line systemic treatment with ipilimumab, an anti-CTLA-4 antibody. Five months later, he developed a subcutaneous metastasis in the left deltoid muscle. Shortly thereafter, he was admitted with a status epilepticus, which resolved upon initiation of antiepileptic drugs. Neither acute hemorrhage nor progression was revealed by brain MRI. A second-line immunotherapy was then initiated with pembrolizumab, an anti-PD-1 antibody at the dose of $2 \mathrm{mg} / \mathrm{kg}$ every 3 weeks. After 18 cycles, a complete metabolic response was obtained on PET scan.

After 22 cycles of pembrolizumab, the patient reported generalized pruritus, partially relieved by a topical steroid cream and oral antihistamine. Eight months later, he developed a new erythematous rash on the trunk. When he came to the clinic 1 month later for the 35th administration of pembrolizumab, we noticed a prurigo-like rash with numerous erythematous erosions on the trunk and limbs and also multiple tense blisters on the palmar face of hands and legs as well as the involvement of oral mucosa (shown in Fig. 1a, b). Punch skin biopsies were performed at the edge of the blisters with the aim to exclude bullous dermatitis. The histopathological examination revealed a complete

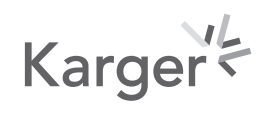




\section{Case Reports in Oncology}
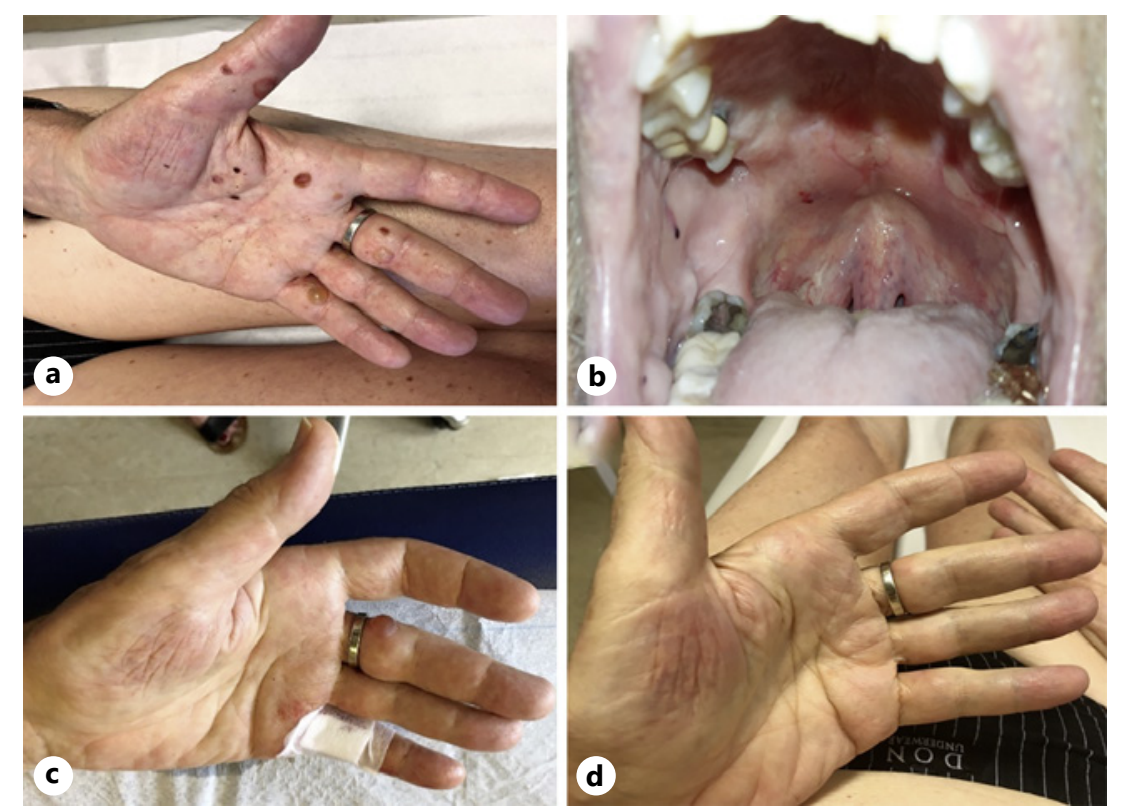

Fig. 1. Clinical presentation of BP induced by pembrolizumab. a Initially at diagnosis with tense blisters on the palmar face of hands. $\mathbf{b}$ Mucosal erosion of the hard palate. c, $\mathbf{d}$ After 4 weeks and after 8 weeks of tapering oral prednisolone. BP, bullous pemphigoid.

detachment of the epidermis with chronic inflammatory infiltrate characterized by eosinophilic granulocytes. Immunochemistry against collagen IV, the main substance located in the lamina densa of basement membrane, showed its attachment to blister base. Direct immunofluorescence showed linear deposition of immunoglobulin G and C3 complement at basement membrane zone (shown in Fig. 2). These histopathological features are consistent with a BP.

Thereafter, we decided to rapidly initiate oral prednisolone $0.2 \mathrm{mg} / \mathrm{kg}$ daily and we permanently discontinued the immunotherapy after a total of 2 years of treatment. Four weeks after the initiation of prednisolone, the patient noticed an improvement of the rash and bullae with partial resolution under tapered dose of prednisolone $4 \mathrm{mg}$ every other day (shown in Fig. 1c). At that moment, considering the absence of complete resolution of BP, we decided to increase dose of prednisolone to $8 \mathrm{mg}$ daily. Four weeks later, all cutaneous lesions had disappeared (shown in Fig. 1d). No BP has reemerged with new tapering regimen.

Unfortunately, only 2 days later, the patient developed an altered state of consciousness with confusion and was admitted to the intensive care unit. He had been compliant with antiepileptic treatment and oral prednisolone. Brain MRI was negative for acute hemorrhage and disease progression, and electroencephalogram was negative for epileptic activity. Lumbar puncture showed a slightly increased cerebrospinal fluid protein concentration at $0.52 \mathrm{~g} / \mathrm{L}$ with normal white blood cell composition and without detection of malignant cells. Autoimmune encephalitis related to pembrolizumab could not be excluded, and this was the reason for the increase of steroid dosage, with progressive improvement of the neurological status. Six months later, a new episode of confusion and epileptic seizure was complicated by a comatose state. No clinical improvement occurred after therapeutic adjustment. In absence of reversibility of the neurological condition, palliative care was initiated, and the patient died 2 months later.

\section{Karger's}




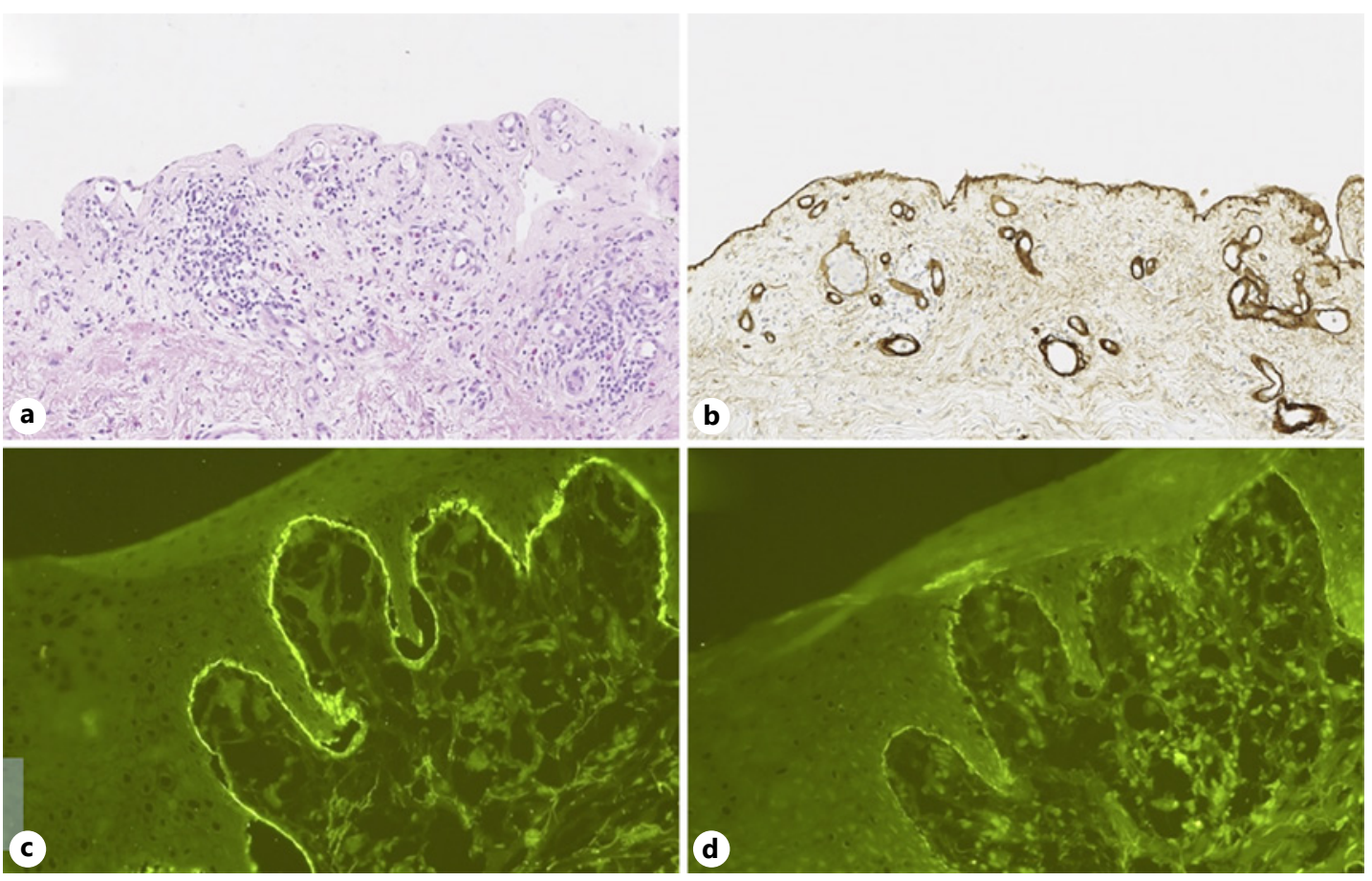

Fig. 2. Microscopic examination of blistered skin punch biopsy. a Complete blistering of the epidermis and an infiltrate of eosinophilic granulocytes in the dermis with persistent dermal papillary festooning in hematoxylin-and-eosin staining. b Persistent collagen IV-positive basal membrane at the floor of the blister in immunohistochemistry staining. c, d Linear deposition of C3 complement and immunoglobulin G at epidermal BMZ under DIF study. DIF, direct immunofluorescence; BMZ, basement membrane zone.

\section{Discussion/Conclusion}

Immune checkpoint inhibition is efficient in metastatic melanoma. Management of irAEs is becoming increasingly challenging due to the wide range of organs potentially involved and of clinical manifestations [3].

$\mathrm{BP}$ is an autoimmune skin disease mainly occurring in the elderly population. The pathogenic mechanism seems to be an autoantibody-mediated immune activation against antigens of the basal membrane of the epidermidis, namely the hemidesmosomal proteins BP180 (mostly NC16a domain) and BP230, resulting in detachment of epidermidis and blister formation. However, blisters may completely be absent in up to $20 \%$ of patients with BP [15, 17]. Multiple studies have investigated the role of T cells in the pathogenesis of BP. Peripheral $\mathrm{T}$ cells are stimulated when exposed to BP180 antigens, resulting in Th2 response and decreased number of T-regulatory cells [10]. Exact mechanisms of ICIs-induced BP are less elucidated: it is possible that inhibition of PD-1 dysregulates B-cell regulatory T cells, resulting in nonspecific production of pathogenic and nonpathogenic autoantibodies [17]. Most of autoantibodies are directed against BP180 NC16A domain, but profiles of the autoantibodies seem involving multiple epitopes [18]. Another evidence has emerged that full-length BP180 protein has been shown to be expressed also in melanoma and could generate autoantibodies against skin [19].

Heterogenous presentation of BP makes the diagnosis challenging. Pruritus may be the predominant symptom of BP and could be present in the prodromal BP phase, but it is also known as one of the most frequent cutaneous irAEs $[3,15,16,20]$. In the case of refractory itch of unknown cause, the hypothesis of BP should be excluded by the investigation of atypical 
clinical features (i.e., excoriations, prurigo, erosions, eczematous, or urticarial lesions) associated with histopathological findings.

Skin biopsy plays a key role in BP diagnosis. Typically, light microscopy findings are inflammatory infiltration of the blister and the dermis with predominant eosinophils. Direct immunofluorescence is the gold standard and shows linear depositions of IgG and/or C3 along the basement membrane zone and sometimes IgA and IgE with a similar pattern $[15,16]$. In our patient, persistent long-term course of pruritus was probably misdiagnosed as low-grade single pruritus irAE and consideration of skin biopsy might have diagnosed the BP at an earlier stage.

Mucosal involvement of BP occurs in around $10-15 \%$ of patients and is associated with disease severity. Oral erosions are the most frequent locations [21]. Our patient presented oral mucosal erosions of the hard palate. No mucosal biopsy was performed given the rapid improvement of oral erosions under systemic steroid therapy.

To date, increasing reports of cases of ICIs-induced BP provide more knowledge about the management of this dermatological entity in cancer patients. In the majority of cases, antiPD-1/PD-L1 antibodies were discontinued, and BP was managed by oral steroids sometimes associated with topical treatments $[7,12,14]$. Sequential therapy of anti-CTLA-4 and antiPD-1 antibodies, as in the case of our patient, may also increase the frequency of cutaneous irAEs in general and of BP in particular.

The particularity of our case is the association of anti-PD-1 induced cutaneous and mucosal $\mathrm{BP}$ with unusual neurological symptoms (recurrent epilepsy, confusion, and coma). Fatal clinical outcome of our patient was secondary to neurological complications without any strong argument of cancer progression. Interestingly, several cases of neurological disorders (e.g., stroke, epilepsy, dementia, and Parkinson's disease) have been described in association with BP and may increase mortality [16, 22-24]. The pathophysiology of this dermatological/neurological association may be explained by the presence of autoantibodies directed against homologous autoreactive antigens (BPAG1 and BPAG2) both in the skin and the brain: this results in cutaneous and neuro-inflammation [22-24]. Therefore, it might be important to perform a close neurological follow-up for BP patients, especially those with a past neurological history.

In conclusion, this is - to our knowledge - the first reported case of a neurological disorder associated to BP in the context of an anti-PD- 1 antibody treatment. The increased use of immunotherapy will likely prompt physicians and health-care professionals to become more familiar with the management of these unusual irAEs.

\section{Statement of Ethics}

The publication of the case was discussed with our institutional Ethics Committee. No ethics approval is required for this case. Given that our patient has died, written informed consent was obtained from the patient's wife for publication of this case report and any accompanying images.

\section{Conflict of Interest Statement}

The authors have no conflicts of interest to declare.

\section{Funding Sources}

I have no funding sources for the publication of this case.

\section{Karger'}




\section{Author Contributions}

Xiaoxiao Wang: selection of the patient for case report, clinical follow-up of the case, and case writing. Mariano Suppa: clinical expertise, performance of the biopsy, and review of the manuscript. Pascal Bruderer: pathological expertise, histopathological images, and review of the manuscript; Nicolas Sirtaine: pathological expertise and review of the manuscript; Sandrine Aspeslagh: clinical expertise and review of the manuscript; Joseph Kerger: clinical follow-up of the case and review of the manuscript.

\section{References}

1 Dine J, Gordon R, Shames Y, Kasler M, Barton-Burke M. Immune checkpoint inhibitors: an innovation in immunotherapy for the treatment and management of patients with cancer. Asia Pac J Oncol Nurs. 2017;4(2):127.

2 Vaddepally RK, Kharel P, Pandey R, Garje R, Chandra AB. Review of indications of FDA-approved immune checkpoint inhibitors per NCCN guidelines with the level of evidence. Cancers. 2020;12(3):738.

3 Brahmer JR, Lacchetti C, Schneider BJ, Atkins MB, Brassil KJ, Caterino JM, et al. Management of immune-related adverse events in patients treated with immune checkpoint inhibitor therapy: American Society of Clinical Oncology Clinical Practice Guideline. J Clin Oncol. 2018;36(17):1714-68.

4 Siegel J, Totonchy M, Damsky W, Berk-Krauss J, Castiglione F, Sznol M, et al. Bullous disorders associated with anti-PD-1 and anti-PD-L1 therapy: a retrospective analysis evaluating the clinical and histopathologic features, frequency, and impact on cancer therapy. J Am Acad Dermatol. 2018;79(6):1081-8.

5 Kuwatsuka Y, Iwanaga A, Kuwatsuka S, Okubo Y, Murayama N, Ishii N, et al. Bullous pemphigoid induced by ipilimumab in a patient with metastatic malignant melanoma after unsuccessful treatment with nivolumab. J Dermatol. 2018;45(1):e21-2.

6 Hanley T, Papa S, Saha M. Bullous pemphigoid associated with ipilimumab therapy for advanced metastatic melanoma. JRSM Open. 2018;9(10):205427041879302.

7 Zumelzu C, Alexandre M, Le Roux C, Weber P, Guyot A, Levy A, et al. Mucous membrane pemphigoid, bullous pemphigoid, and anti-programmed death-1/programmed death-ligand 1: a case report of an elderly woman with mucous membrane pemphigoid developing after pembrolizumab therapy for metastatic melanoma and review of the literature. Front Med. 2018;5:268.

8 Haug V, Behle V, Benoit S, Kneitz H, Schilling B, Goebeler M, et al. Pembrolizumab-associated mucous membrane pemphigoid in a patient with Merkel cell carcinoma. Br J Dermatol. 2018;179(4):993-4.

9 Parakh S, Nguyen R, Opie JM, Andrews MC. Late presentation of generalised bullous pemphigoid-like reaction in a patient treated with pembrolizumab for metastatic melanoma. Australas J Dermatol. 2017;58(3):e10912.

10 Mochel MC, Ming ME, Imadojemu S, Gangadhar TC, Schuchter LM, Elenitsas R, et al. Cutaneous autoimmune effects in the setting of therapeutic immune checkpoint inhibition for metastatic melanoma: cutaneous autoimmune effects of checkpoint inhibitors. J Cutan Pathol. 2016;43(9):787-91.

11 Lopez AT, Geskin L. A case of nivolumab-induced bullous pemphigoid: review of dermatologic toxicity associated with programmed cell death protein-1/programmed death ligand-1 inhibitors and recommendations for diagnosis and management. Oncologist. 2018;23(10):1119-26.

12 Lopez AT, Khanna T, Antonov N, Audrey-Bayan C, Geskin L. A review of bullous pemphigoid associated with PD-1 and PD-L1 inhibitors. Int J Dermatol. 2018;57(6):664-9.

13 Hwang SJE, Carlos G, Chou S, Wakade D, Carlino MS, Fernandez-Penas P. Bullous pemphigoid, an autoantibodymediated disease, is a novel immune-related adverse event in patients treated with anti-programmed cell death 1 antibodies. Melanoma Res. 2016;26(4):413-6.

14 Anastasopoulou A, Papaxoinis G, Diamantopoulos P, Christofidou E, Benopoulou O, Stratigos A, et al. Bullous pemphigoid-like skin lesions and overt eosinophilia in a patient with melanoma treated with nivolumab: case report and review of the literature. J Immunother. 2018;41(3):164-7.

15 Feliciani C, Joly P, Jonkman MF, Zambruno G, Zillikens D, Ioannides D, et al. Management of bullous pemphigoid: the European Dermatology Forum consensus in collaboration with the European Academy of Dermatology and Venereology. Br J Dermatol. 2015;172(4):867-77.

16 Ujiie H, Iwata H, Yamagami J, Nakama T, Aoyama Y, Ikeda S, et al. Japanese guidelines for the management of pemphigoid (including epidermolysis bullosa acquisita). J Dermatol. 2019;46(12):1102-35.

17 Singer S, Nelson CA, Lian CG, Dewan AK, LeBoeuf NR. Nonbullous pemphigoid secondary to PD-1 inhibition. JAAD Case Rep. 2019;5(10):898-903.

18 Aoki Y, Miyagawa F, Miyashita K, Nishimura Y, Hashimoto T, Asada H. Nivolumab-associated bullous pemphigoid reactions involving multiple epitopes. Eur J Dermatol. 2019;29(5):552-4.

19 Krenacs T, Kiszner G, Stelkovics E, Balla P, Teleki I, Nemeth I, et al. Collagen XVII is expressed in malignant but not in benign melanocytic tumors and it can mediate antibody induced melanoma apoptosis. Histochem Cell Biol. 2012;138(4):653-67. 
20 Sibaud V. Dermatologic reactions to immune checkpoint inhibitors: skin toxicities and immunotherapy. Am J Clin Dermatol. 2018;19(3):345-61.

21 Kridin K, Bergman R. Assessment of the prevalence of mucosal involvement in bullous pemphigoid. JAMA Dermatol. 2019;155(2):166.

22 Försti AK, Jokelainen J, Ansakorpi H, Seppänen A, Majamaa K, Timonen M, et al. Psychiatric and neurological disorders are associated with bullous pemphigoid: a nationwide Finnish Care Register study. Sci Rep. 2016; 6(1):37125.

23 Milani-Nejad N, Zhang M, Kaffenberger J. The association between bullous pemphigoid and neurological disorders: a systematic review. Eur J Dermatol. 2017;27(5):472-81.

24 Tarazona MJ, Mota AN, Gripp AC, Unterstell N, Bressan AL. Bullous pemphigoid and neurological disease: statistics from a dermatology service. An Bras Dermatol. 2015;90(2):280-2. 